\title{
One-Way Two-Color Fiber Link for Frequency Transfer
}

\author{
J. L. Hanssen, S. G. Crane, and C. R. Ekstrom \\ Time Service Department \\ US Naval Observatory \\ Washington, DC USA \\ james.hanssen@usno.navy.mil
}

\begin{abstract}
We discuss our work on two-color one-way frequency transfer over optical fiber. We have expanded on our previous work by extending the wavelength separation by using one channel in the $C$ band at $1553 \mathrm{~nm}$ and the other channel in the $O$ band at $1310 \mathrm{~nm}$. Both wavelengths have low loss and are well suited to use in optical fiber networks. We present measurements of the improved two-color phase delay as a function of temperature and compare that to the actual phase delay brought about by temperature induced length changes in the link. We also show improved closed loop performance of the link, including the dependence on the total length of the link.
\end{abstract}

\section{INTRODUCTION}

There has been great progress in clock stability and accuracy in the past years. Microwave clocks based on atomic fountain technology have achieved short term stabilities in the upper $10^{-14} \mathrm{~s}$ integrating down into the low $10^{-16} \mathrm{~s}$ in a day [1]. Optical clocks have improved upon that and have short term stabilities in the low $10^{-15} \mathrm{~s}$ at a second averaging down into the $10^{-17} \mathrm{~s}$ in an hour [2]. It is important to have high quality transfer techniques to take advantage of this improved performance.

Fiber optic links have recently been shown to be an excellent candidate for high quality time and frequency transfer. The highest performance links are two-way and require bidirectional access to the fiber. The stable frequency is encoded on the light either through amplitude modulation or directly through the phase of the light. Due to the low losses in optical fiber, the signal can travel hundreds of kilometers before reaching a receive station where the signal is recovered. A small portion of the transmitted signal is returned to the sending station along the same fiber where any phase fluctuations imparted by the fiber are then measured and appropriately compensated for. For amplitude modulated schemes, short term stabilities of $10^{-15}$ integrating down into the low $10^{-18} \mathrm{~s}$ have been demonstrated [3-4]. For coherent carrier schemes involving the phase of the light, better performance has been reached with short term stabilities in the $10^{-16} \mathrm{~s}$ averaging down to the $10^{-19} \mathrm{~s}$ in a day [4-5].
While two-way links have excellent performance, there is great interest in one-way transfer links with similar performance. One-way links use a proxy to determine the fiber induced phase fluctuations as opposed to directly measuring them. There are several advantages to using a oneway technique. Two-way methods are necessarily invasive and require access to the fiber to install bidirectional amplifiers and other specialized equipment. A one-way technique would work well with the inherent unidirectional nature of modern fiber optic networks. Two-way methods also are rather cumbersome from a network topology standpoint. For every receive station that wants to obtain a copy of the transmitted frequency, a two-way link must be established. A one-way link offers the possibility of a simpler broadcast topology. The send station can passively broadcast the high quality signal and each receive station can appropriately compensate and recover the high quality frequency.

In the work presented here, we expand on our previous work on two-color, one-way optical fiber links [6]. In that system, a two-color signal was used as a proxy for temperature induced phase changes in the fiber. By using two colors in the $\mathrm{C}$ band, we were able to show the sensitivity of the two-color signal to phase changes caused by temperature and we were able to demonstrate a closed compensated link for frequency transfer. To improve upon that work, we have used two colors with a larger separation in wavelength. It was found to have improved sensitivity and performance.

\section{BACKGROUND}

In order to have a one-way link, it is necessary to have a monitor of the fiber induced phase fluctuations. For phase shifts due to temperature induced expansion in the fiber, the two-color signal provides this proxy [7]. The two-color scheme relies on the fact that there is dispersion in the fiber and that the dispersion is temperature dependent. Suppose that a microwave source is split and used to intensity modulate two different colors that are sent down the same fiber. At the far end of the fiber, the colors are separated and the

Funding support from DARPA QuASAR Program and SPAWAR 
microwave signal on each color is recovered. If the signals are compared, there will be phase difference between the two signals, because the dispersion gives each color a different effective path length to travel. Now, if the temperature of the fiber changes, the index of refraction will change, but it changes differently for the two colors. Therefore, on the far end, the phase difference between the two microwave signals will change as the temperature changes. This gives a proxy signal for the temperature induced phase fluctuations in the fiber which can be used to cancel the phase excursions of either of the transmitted microwave signals

We model this using temperature dependent Sellmeier coefficients for fused silica [8]. The temperature dependent index of refraction has this form

$$
n^{2}(\lambda, T)-1=\sum_{i=1}^{3} \frac{S_{i}(T) \cdot \lambda^{2}}{\lambda^{2}-\lambda_{i}^{2}(T)}
$$

The $S_{i}$ coefficients are determined by the strength of resonance features that occur within the fiber at wavelengths $\lambda_{i}$. A thorough description of this model as well as the complete set of values for the different coefficients can be found in [8].

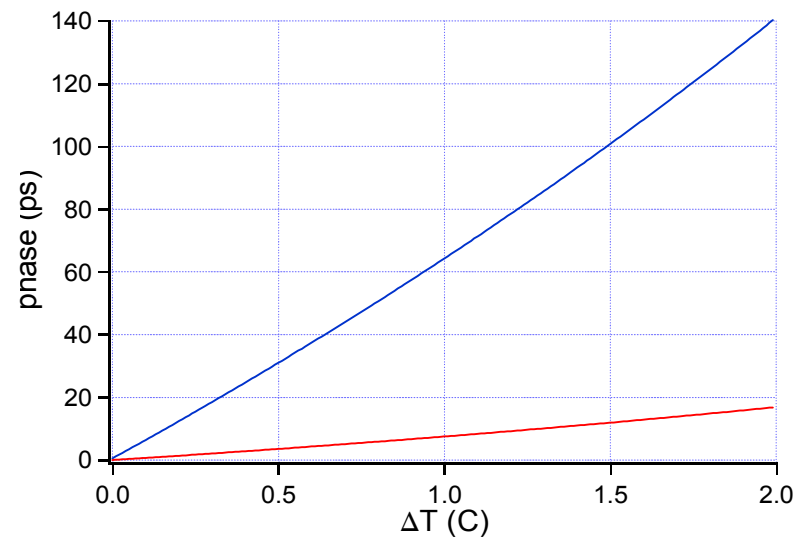

Figure 1. Calculation of the time delay between two signals transmitted on fiber at two separate wavelengths as a function of temperature difference. The red corresponds to $1530 \mathrm{~nm}$ and $1553 \mathrm{~nm}$. The blue corresponds to $1310 \mathrm{~nm}$ and $1553 \mathrm{~nm}$. The calculation was done for $1 \mathrm{~km}$ of fiber.

Using this model, the phase shift between signals sent on two different colors can be determined. Figure 1 shows the result of that model for two different pairs of colors. For a $1 \mathrm{~km}$ length of fiber, the time delay between two signals at two different colors was calculated as a function of temperature change in the fiber. The red curve corresponds to two colors in the C band, $1530 \mathrm{~nm}$ and $1553 \mathrm{~nm}$, that were used in our previous work [6]. The blue curve is for one color in the $\mathrm{C}$ band, $1553 \mathrm{~nm}$, and one color in the $\mathrm{O}$ band, $1310 \mathrm{~nm}$. As can be seen, there is a much greater time delay for the two colors with the larger wavelength separation. There is a factor of approximately 8.5 greater sensitivity to temperature induced phase changes in the fiber over our previous results which should lead to better compensated link performance.

\section{EXPERIMENT}

Our previous experimental setup was adjusted to investigate the potential improved performance. A brief description of this setup follows. A high quality $5 \mathrm{MHz}$ crystal oscillator is multiplied up to $6.8 \mathrm{GHz}$. That signal is split and used to modulate the intensity of two fiber coupled laser diodes, one at $1553 \mathrm{~nm}$ and one at $1310 \mathrm{~nm}$. The output of each diode passes through a polarization scrambler before being combined with an add/drop filter. The light then travels down the fiber under test, usually a spool of fiber ranging from $1 \mathrm{~km}$ to $50 \mathrm{~km}$. The light passes to a temperature controlled spool that has $5 \mathrm{~km}$ of fiber. The two colors are separated with an add/drop filter and the microwave signals are recovered. A mixer is used to recover the relative phase between the two colors. This is the two-color signal mentioned earlier. In addition, for one of the colors, a portion of the microwave signal is split off and used to phase lock a $5 \mathrm{MHz}$ crystal to the transmitted microwave signal. This provides a high quality RF output on the receive side of the link. For a more detailed description see reference [6].

The two colors used in our earlier work were in the C band: $1530 \mathrm{~nm}$ and $1553 \mathrm{~nm}$. With this setup, we were able to characterize the two-color link. We performed open loop measurements to determine the ratio of the temperature induced phase shift on the transmitted microwave signal to the phase shift of the two-color signal, also called the lever arm. For the two colors in the $\mathrm{C}$ band, the lever arm was approximately 320 . This value is important because it determines the noise properties of the locked link. The twocolor signal will be multiplied up by the lever arm to compensate the phase fluctuations. Therefore, any noise on the two-color signal will also be multiplied up by the lever arm and added to the transmitted signal.

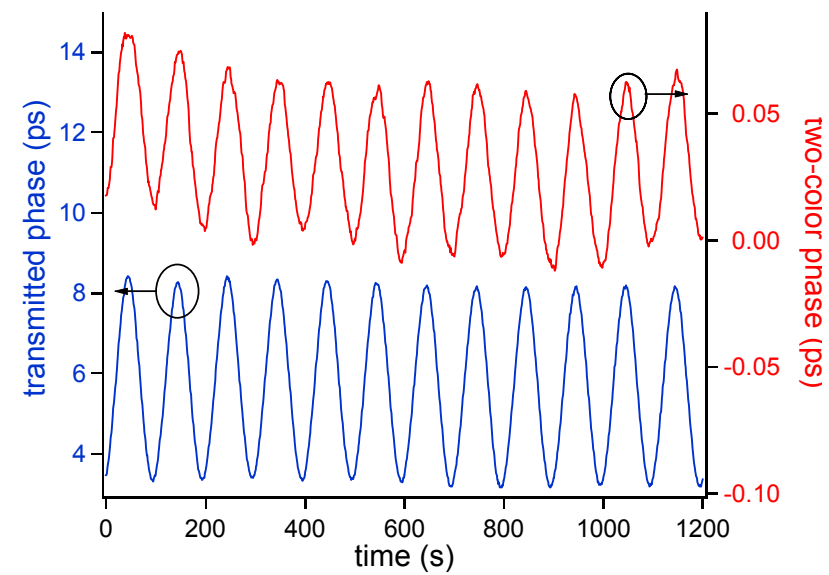

Figure 2. Comparison of the actual temperature induced phase change in the transmitted signal and the two-color signal. The blue curve is the relative phase difference between the RF signal on the send side and the RF signal on the receive side as the temperature of the fiber is cycled. The red color is the two-color signal while the temperature of the fiber is cycled. The horizontal axis is time in seconds.

The same open loop tests were done using the $\mathrm{O}$ band and $\mathrm{C}$ band light. A $5 \mathrm{~km}$ spool of fiber was used as the fiber 
under test and placed in an environmental chamber that varied the temperature by approximately five degrees Celsius. The two-color signal was monitored as well as the relative phase of the RF signals on the send and receive sides. Figure 2 shows the results of that measurement. It is clear that the two-color signal tracks the relative phase signal quite well. There are small additional low frequency movements in the two-color signal due to small out-of-loop effects. The lever arm of this system is approximately 85 . This is almost a factor of four improvement over the previous results using colors in the $\mathrm{C}$ band, but as good as the 8.5 predicted by the simulations.

Since the two-color noise is multiplied by the lever arm and written on the transferred signal when the link is locked, it is important to understand the noise properties of the twocolor signal. Previously, it was shown that the two-color noise grew linearly with length. This was verified for the new combination of colors. The two-color signal was recorded and analyzed for varying lengths of fiber. The results of these measurements are shown in Figure 3 along with our previous results. The noise grows linearly with distance as before. What is interesting to note is that the slope is increased over the previous results. This shows that for larger wavelength separations, there is increased noise that is added to the transmitted signal. To test that this effect is actually due to a length dependent effect and not simply attenuation of the signal, a $10 \mathrm{~m}$ cable with a variable attenuator was used between the send and receiver side. The attenuation was set such that it was the same as a $35 \mathrm{~km}$ length of fiber. The noise was what was expected of a $10 \mathrm{~m}$ fiber and is shown as a green dot in Figure 3.

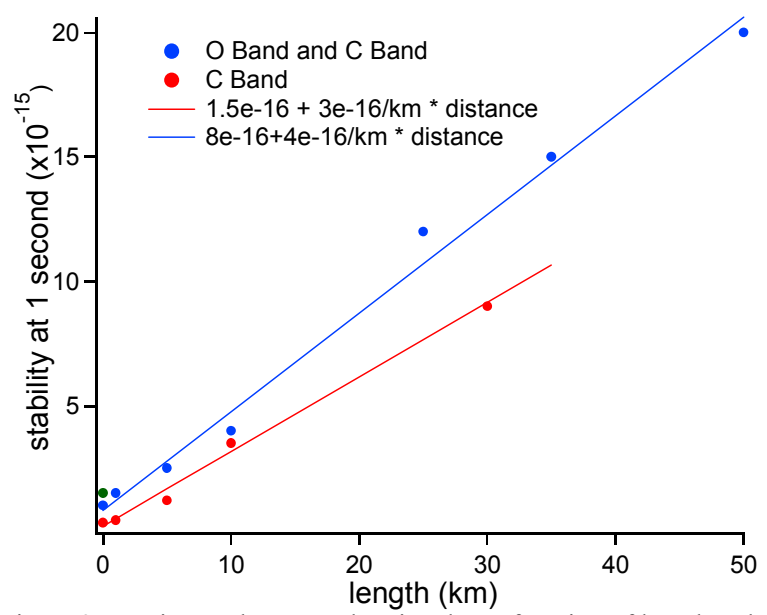

Figure 3. Noise on the two-color signal as a function of length. The red curve corresponds to the 1 second stability of the two color signal for two colors in the $\mathrm{C}$ band. The blue curve corresponds to the 1 second stability of the two color signals with one color in the $\mathrm{O}$ band and one color in the $\mathrm{C}$ band. The solid lines are fits to the data.

Based on the previous two measurements, it is clear that it should be possible to use the two-color signal as an error signal to compensate for temperature induced phase changes. In order to confirm this, a $10 \mathrm{~km}$ test link was built in the lab.
The link consisted of two $5 \mathrm{~km}$ spools of SMF28. The first spool was placed in an environmental chamber. The chamber allowed for coarse adjustment of the overall temperature of the fiber. The second spool was in an environmentally isolated foam container with fine temperature control capabilities. This spool was used for compensation in closed loop tests. The first test was to observe the open loop performance of the link. The temperature controlled spool was held constant while the test spool was put through a series of temperature changes designed to produce flicker noise. Figure 4 shows the Allan deviation of the transmitted signal of this unlocked link. The stability initially integrates down as $1 / \tau$ until the effects of varying the temperature start to degrade the stability. The stability increases to the $10^{-13}$ level and flickers.

The next test was to see the effect of using the two-color signal to compensate for temperature induced phase fluctuations. The test spool was put through the same temperature cycling regimen, but this time, a PI circuit was used to drive the temperature of the compensation spool to null out the two-color signal. The results are shown in Figure 4. There is a clear suppression of the temperature induced phase noise. The stability initially follows the same trace as the unlocked data. Around the time constant for the servo loop, which is a few tens of seconds, the Allan deviation starts to integrate down as $1 / \tau^{2 / 3}$ just as the two-color signal noise does. At $10^{4}$ seconds, there is over an order of magnitude improvement in the stability as compared to the uncompensated link.

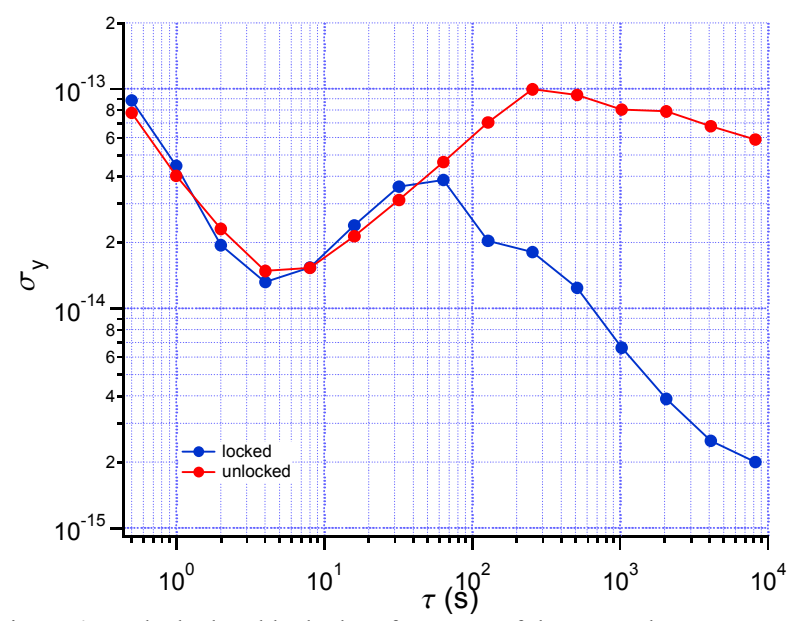

Figure 4. Unlocked and locked performance of the two-color, one-way link. The red trace corresponds to the stability of the transmitted RF for an uncompensated link. The blue trace corresponds to the stability of the transmitted RF when the two-color signal is used to actively compensate for phase fluctuations due to temperature changes.

Figure 5 shows a comparison between our previous work using wavelengths only in the $\mathrm{C}$ band and the work presented here using wavelengths in the $\mathrm{O}$ band and $\mathrm{C}$ band. The results are both for $10 \mathrm{~km}$ links utilizing the two-color signal to compensate for phase fluctuations induced by temperature. It can immediately be seen that by moving to a larger 
wavelength separation, there was an improvement in the performance of the two-color lock by a factor of two. Our original work had a long term behavior of $1 \times 10^{-12} / \tau^{2 / 3}$. That has been improved by a factor of two to $5 \times 10^{-13} /$ $\tau^{2 / 3}$.

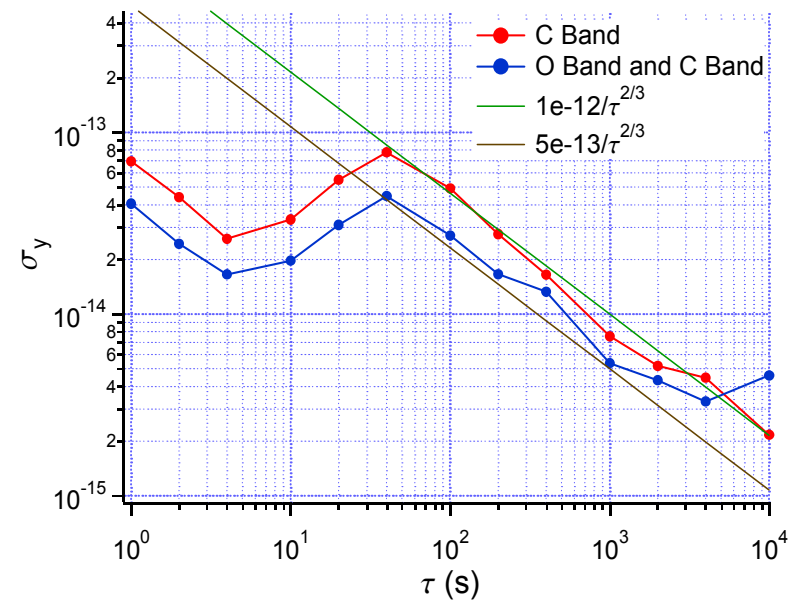

Figure 5. Comparison of the previous two-color link results with the present results. The red trace is the previous result and the blue trace is the result presented here. The straight lines show the long term behavior of the links. For the $\mathrm{C}$ band only link, the long term behavior integrates down as $1 \times 10^{-12} / \tau^{2 / 3}$. For the $\mathrm{O}$ band and $\mathrm{C}$ band, the long term behavior integrates down as $5 \times 10^{-13} / \tau^{2 / 3}$.

It should be noted that while using a larger wavelength separation improved the performance of the locked link, it was not as great as initially anticipated. Several issues brought this about. The initial calculations suggested a factor of 8.5 improvement in the lever arm, yet measurements revealed the improvement to be only a factor of 4 . This discrepancy can be attributed to the limitations of the temperature dependent Sellmeier coefficient model. That aside, the factor of four improvement in the lever arm could not be fully realized as well. The larger wavelength separation brought an increase in the noise on the two-color signal, which degraded the final performance of the locked link. Still, there was a net improvement in the performance of the link.

\section{CONCLUSIONS}

In conclusion, we have demonstrated a two-color, one-way link for high quality frequency transfer. We have increased the wavelength separation from our previous work by using wavelengths in the $\mathrm{O}$ band and the $\mathrm{C}$ band. This has led to an improvement of the sensitivity of the two-color signal to temperature induced phase changes, but it also led to increased noise on the two-color signal. The net result of this was an improvement by a factor of two over previous efforts to create a compensated one-way link. This technique clearly holds promise for high quality frequency transfer especially in situations where bidirectional fiber access is not possible.

[1] R. Wynands and S. Weyers, "Atomic Fountain Clocks," Metrologia, vol. 42, pp. S64-S79, 2005.

[2] A. D. Ludlow, et. al. , "Sr Lattice Clock at $1 \times 10^{-16}$ Fractional Uncertainty by Remote Optical Evaluation with a Ca Clock," Science, vol. 319, pp. 1805-1808, 2008.

[3] O. Lopez, A. Amy-Klein, M. Lours, C. Chardonnet, and G. Santarelli, "High-Resolution Microwave Frequency Dissemenation on an $86 \mathrm{~km}$ Urban Optical Link," App. Phys. B, vol. 98, pp. 723-727, 2009.

[4] S. M. Foreman, K. W. Holman, D. D. Hudson, D. J. Jones, and J. Ye, "Remote Transfer of Ultrastable Frequency References via Fiber Networks," Rev. Sci. Instrum., vol. 78, p. 021101, 2007.

[5] H. Jiang, et. al. "Long-Distance Frequency Transfer Over an Urban Fiber Link Using Optical Phase Stabilization," JOSA B, vol. 25, pp. 2029-2035, 2008.

[6] J. L. Hanssen, S. G. Crane, and C. R. Ekstrom, "One-Way Temperature Compensated Fiber Link," Proceedings of the Frequency Control and the European Time and Frequency Forum (FCS), 2011 Joint Conference of the IEEE International, 2011.

[7] S.-C. Ebenhag, P. O. Hedekvist, and K. Jaldehag, "Fiber-Based Frequency Distribution Based on Long-Haul Communication Lasers," $41^{\text {st }}$ Annual Precise Time and Time Interval Meeting, 2009.

[8] B. B. Leviton and B. J. Frey," Temperature-Dependent Absolute Refractive Index Measurements of Synthetic Fused Silica," Proc. SPIE 6273, 6273K, 2006. 